\title{
Extrapineal mature teratoma of the posterior fossa in a child
}

\author{
Mónica Rivero-Garvía, Javier Márquez-Rivas, Eloy Rivas, Ana Belén Rueda-Torres
}

Mónica Rivero Garvía, Division of Pediatric Neurosurgery, Hospital Virgen del Rocío, Manuel Siruot s/n, Seville, Spain Email: monicargarvia@msn.com

Received 13 July 2011; revised 21 September 2011; accepted 19 July 2012

\begin{abstract}
Introduction: Teratomas are non-malignantgerm cell tumors commonly composed of cell types derived from all of the three germ layers. Intracerebral teratomas typically are midline or paraxial lesions located in the pituitary stalk or the pineal region. We report our experience with mature teratomas of the posterior fossa. Case report: We present the case of an 11-yearold caucasian female with progressive headache that caused interrupted sleep. Cerebral magnetic resonance imaging showed a midline lesion in the posterior fossa with mass effect and without contrast enhancement. Anatomic pathology revealed a mature teratoma. Conclusion: Differential diagnosis of midline lesions in pediatric patients must include teratomas in spite of being posterior fossa lesions.
\end{abstract}

Keywords: Mature Teratoma; Posterior Fossa; Child; Gross Total Reseccion; Extrapineal

\section{INTRODUCTION}

Teratomas are neoplasms that contain tissues derived from the three germ layers: ectoderm (e.g. skin and hair), mesoderm (e.g. adipose tissue), and endoderm (e.g. respiratory structures and digestive organs). Histologically, these lesions are classified as something between germcell tumors and dysembryoplastic neuroepithelial lesions. In contrast to teratomas, epidermoid cysts are the lesions derived from a single layer, that is, the ectoderm (e.g keratin), while dermoid cysts are the ones derived from two layers, the ectoderm and the mesoderm. The presence of adipose tissue in these latter tumors constitutes the main difference between them [1-4].

With a clear predominance in males, teratomas account for $0.2 \%$ of all intracranial tumors, although this incidence increases to $2 \%$ in the first two decades of life [1-3].

In this paper, we present the exceptional case of a pure extrapineal mature teratoma of the posterior fossa.

\section{CASE REPORT}

We report the case of an 11-year-old Caucasian female that presented with progressive headache that caused interrupted sleep. Non-contrast computed tomography (CT) of the head revealed a well circumscribed, hypointense lesion in the midline posterior fossa without accompanying edema (Figure 1). Contrast magnetic resonance imaging (MRI) showed an extra-axial lesion in the midline posterior fossa with a mass effect that caused the displacement of the vermis and the cerebral hemispheres.

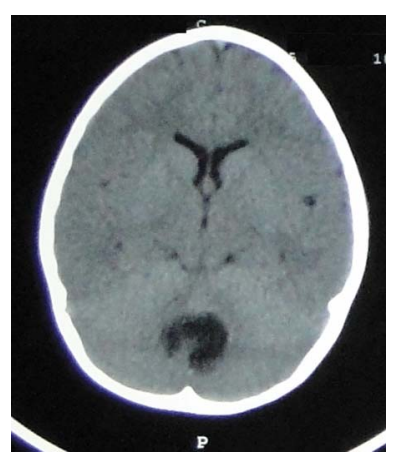

(a)

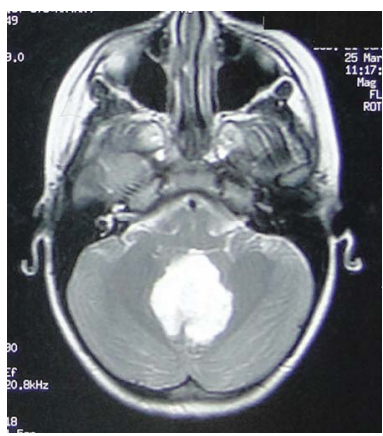

(c)

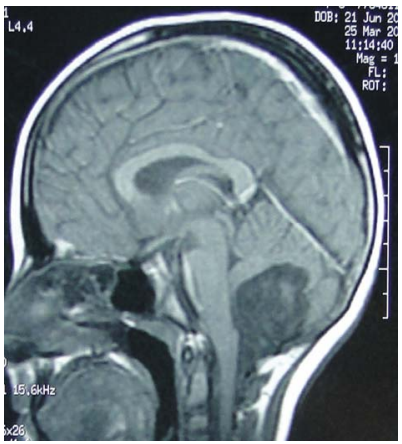

(b)

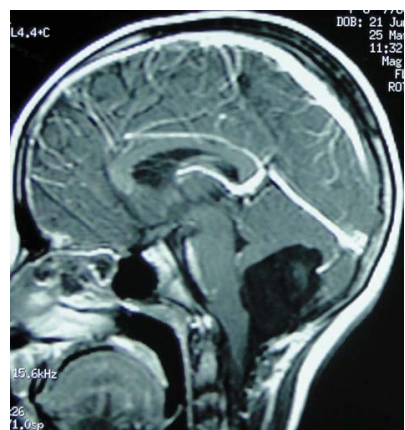

(d)
Figure 1. (a) Non-contrast axial CT scan of the head showing a hypodense lesion in the posterior fossa in absence of associated hydrocephalus; (b) Sagital T1-weighted image without contrast revealing a hypointense tumor in the posterior fossa; (c) Sagittal T2-weighted image showing a hyperintense lesion without accompanying edema; (d) Contrast sagital T1-weighted showing a lesion that does not enhance after contrast infusion. 
The lesion was hypointense on T1-weighted images and hyperintense on T2-weighted studies, and showed no enhancement after contrast administration. The main diagnosis was epidermoid cysts. The patient underwent suboccipital craniotomy (Figure 1). After V-shaped dural opening, the tumor was explored, revealing the presence of desquamated keratin as well as hair follicles surrounded by a thin capsule (Figure 2). Gross total resection of the lesion was achieved.

The patient was not sequelae of the disease.

Histological study revealed components of all three germ layers (e.g. keratin cyst formation, stratified squamous keratinized epithelium, hair follicles, bone or adipose tissue) (Figure 3). As a result, a mature teratoma was finally diagnosed without malignant microfoci.

Alpha-fetoprotein and beta-HCG were not determined preoperative because suspected diagnosis were epidermoid cysts, neither postoperative because there were

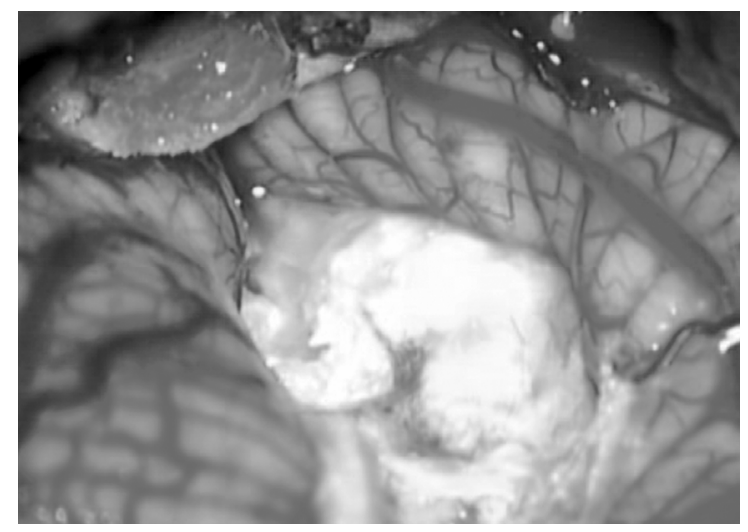

Figure 2. Intraoperative image of the tumor showing desquamated keratin and hair follicles.

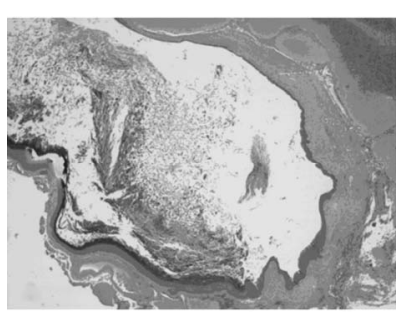

(a)

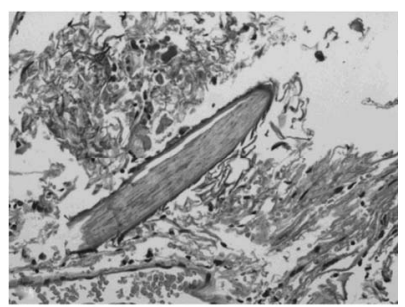

(c)

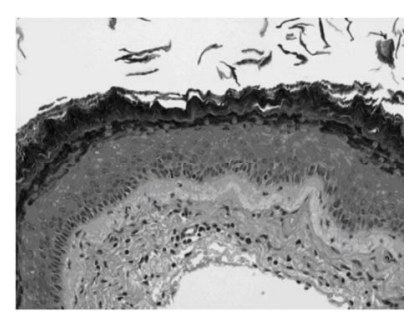

(b)

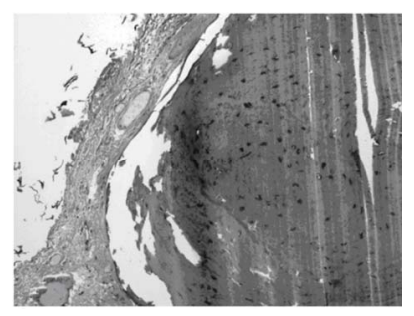

(d)
Figure 3. Anatomic pathology studies. (a) Keratin cyst formation; (b) Stratified squamous keratinized epithelium; (c) Hair; (d) Bone. malignant microfoci and abdominal-CT did not show other teratoma.

\section{DISCUSSION}

Intracranial mature teratomas are tumors with a very low incidence $(0.2 \%)$ and a clear male predominance $(5: 1)$. This incidence is higher in pediatric patients (2\%). Intracranial teratomas usually are located in the supratentorial midline and the pineal region [1-3]. We report the case of an 11-year-old Caucasian female with a mature teratoma in the midline posterior fossa.

Definitive diagnosis is achieved by means of a histological study, when tissues derived from the three germ layers are identified.

Although no radiological alteration specific of these lesions has been described, the presence of midline lesions in pediatric patients must take clinicians raise a high index of suspicion of teratoma $[2,5,6]$.

To our knowledge, only three cases of posterior fossa mature teratomas have been reported in detail in the literature to date. However, they all present the cases of adult patients and only one of them is a midline teratoma [1], being the other two ones paramedian. Further cases on this matter describe the mature component in association with immature tissue, other germ tumors, or as a consequence of extra-cranial lesions.

When reviewing the literature, the case series of intracranial pediatric teratomas reported by Noudel et al. revealed pure mature teratomas in 5 out of 14 patients with pure mature teratomas [2]. However, all of them were located in the sellar or the pineal regions and none of them in the posterior fossa. Kong et al. reported 6 cases of teratoma from a total case series of 36 patients. Within these 6 cases, only one was settled in the posterior fossa and all of them presented with a mixed histology, where the mature teratoma was only part of the lesion [5]. On the contrary, our case describes a lesion that histologically constitutes a pure mature teratoma. In larger series of pediatric tumors, such as the ones reported by Rashidi M. et al., teratomas are not even described [6].

In view of all these findings, we can now conclude that this is the first case of midline posterior fossa mature teratoma in a child published in the literature to date.

Complete surgical excision is the most effective treatment in cases of benign intracranial lesions with mass effect, and enables the patient's cure. Other alternatives, such as local radiotherapy, can cause a sudden increase in the lesion size-as evidenced by Kong et al. [5], as well as a higher risk of secondary neoplasms in pediatric patients.

\section{CONCLUSIONS}

Differential diagnosis of midline lesions in pediatric pa- 
tients must include teratomas in spite of being posterior fossa lesions.

Surgery is the main therapeutic approach and a complete surgical resection is usually necessary to offer potentially curative therapy to patients.

A definitive diagnosis is provided by histopathological study when the presence of tissues derived from the three germ layers is demonstrated.

\section{REFERENCES}

[1] Beschorner, R., Schittenhelm, J., Bueltmann, E., et al. (2009) Mature cerebellar teratoma in adulthood. Neuropathology, 29, 176-180. doi:10.1111/j.1440-1789.2008.00940.x

[2] Noudel, R., Vinchon, M., Dhellemmes, P., et al. (2008) Intracranial teratomas in children: The role and timing of surgical removal. Journal of Neurosurgery: Pediatrics, 2, 331-338. doi:10.3171/PED.2008.2.11.331

[3] Drapkin, A.J., Rose, W.S. and Pellmar, M.B. (1987) Mature teratoma in the fourth ventricle of an adult: Case report and review of the literature. Neurosurgery, 21, 404410. doi:10.1227/00006123-198709000-00024

[4] Zavanone, M., Alimehmeti, R., Campanella, R., et al. (2002) Cerebellar mature teratoma in adulthood. Journal of Neurosurgical Sciences, 46, 35-38.

[5] Kong, D.-S., Nam, D.-H., Lee, J.-I., et al. (2009) Intracranial growing teratoma syndrome mimicking tumor relapse: A diagnostic dilemma. Journal of Neurosurgery: Pediatrics, 3, 392-396. doi:10.3171/2009.1.PEDS0857

[6] Rashidi, M., DaSilva, V.R., Minagar, A., et al. (2003) Nonmalignant pediatric brain tumors. Current Neurology and Neuroscience Reports, 3, 200-205. doi:10.1007/s11910-003-0079-9 